\title{
Time of maximum cycloplegia after instillation of cyclopentolate $1 \%$ in children with brown irises
}

\author{
This article was published in the following Dove Press journal: \\ Clinical Ophthalmology \\ 18 May 2016 \\ Number of times this article has been viewed
}

\section{Sittikorn Laojaroenwanit \\ Vimontip Layanun \\ Pokpong Praneeprachachon \\ Parnchat Pukrushpan}

Department of Ophthalmology, Faculty of Medicine, Chulalongkorn

University, Bangkok, Thailand
Correspondence: Parnchat Pukrushpan Pediatric Ophthalmology and Strabismus Division, Department of Ophthalmology, Faculty of Medicine, Chulalongkorn University, 1873 Rama 4 Road, Pathumwan, Bangkok 10330, Thailand

Tel +6622564142

Fax +6622528290

Email parnchat@gmail.com
Purpose: We aimed to 1) determine the time of maximum cycloplegia after instillation of cyclopentolate $1 \%$ in children with brown irises, 2) evaluate the correlation between the pupillary reaction and time of maximum cycloplegia, and 3) identify any side effects of the medication.

Patients and methods: This was a prospective analytical study involving children aged 5 to 14 years who were attending refraction clinic. Cyclopentolate $1 \%$ was instilled three times at 10 -minute intervals. The spherical equivalent, pupillary reaction, and pupillary diameter were recorded before the first drop and nine times after the last drop at 10-minute intervals. Side effects were assessed. Time of maximum cycloplegia was determined from the time point at which the $95 \%$ confidence interval of the differences between the mean spherical equivalent at each point and its final value at 110 minutes was reached and remained within the equivalence limit $( \pm 0.25 \mathrm{D})$.

Results: Sixty children were enrolled in this study. Their mean age was 9.8 years (range: 5-14 years). Time of maximum cycloplegia was reached at 30 minutes after the first instillation of cyclopentolate. A poor correlation was observed between the pupillary reaction and the time of maximum cycloplegia $(r=-0.07)$. The mean pupillary diameter at 30 minutes was $3.7 \pm 1.3 \mathrm{~mm}$, and further dilation occurred thereafter. No side effects were observed.

Conclusion: In most children, maximum cycloplegia was reached 30 minutes after the first instillation of cyclopentolate. The absence of a pupillary reaction should not be used as an indicator of maximum cycloplegia.

Keywords: refraction, cyclopentolate, cycloplegia, brown irises, cycloplegic refraction

\section{Introduction}

Maximum cycloplegia is necessary for accurate measurement of refractive error in children because it maximally inhibits accommodation and thereby prevents overestimation of myopia and underestimation of hyperopia. ${ }^{1}$ Cyclopentolate $1 \%$ is one of the most frequently used cycloplegic agents in children due to its low incidence of systemic reactions and rapid onset of cycloplegia.

The time of maximum cycloplegia has been found to vary from 10 to 60 minutes after instillation of cyclopentolate. ${ }^{2-7}$ The timing has also been reported to show a large difference among adults with different iris colors. ${ }^{6}$ Other than the studies by Lin et $\mathrm{al}^{7}$ and Wang et al, ${ }^{8}$ who reported the time of maximum cycloplegia in myopic Taiwanese and hyperopic Chinese children, there is currently no other evidence on the time of maximum cycloplegia in Asian children with brown irises.

In clinical practice, most ophthalmologists wait until the pupils are fully dilated or unresponsive to light before performing cycloplegic refraction. This could result in unnecessary long waiting time or measurement error if maximum cycloplegia occurs 
before or after the maximum pupillary dilatation or the absence of pupillary reaction developed.

In this prospective descriptive study, we aimed to 1) determine the time of maximum cycloplegia after instillation of cyclopentolate $1 \%$ in children with brown irises, 2 ) evaluate the correlation between the time of maximum cycloplegia and the pupillary reaction, and 3) identify any serious side effects of the medication.

\section{Patients and methods}

This study followed the World Medical Association Declaration of Helsinki. The research protocol was approved by the Institutional Review Board of King Chulalongkorn Memorial Hospital, Chulalongkorn University (IRB number 001/56) and registered to the Thai Clinical Trial Registry (TCTR20140219001). Written informed assent and informed consent were obtained from all the patients and their parents.

Thai children aged 5 to 14 years attending the refraction clinic at King Chulalongkorn Memorial Hospital from July 2013 to June 2014 were enrolled in this study. The exclusion criteria included ophthalmic diseases other than refractive error and/or strabismus, a history of ophthalmic surgery, a history of allergy to cyclopentolate, known cardiovascular disease, and insufficient cooperation that would interfere with instillation of eye drops or outcome measurements.

All patients underwent refraction and measurement of their pupillary reaction and pupillary diameter before instillation of the eye drops. They then underwent instillation of one drop of $0.5 \%$ tetracaine eye drops (Alcon, Inc., Hünenberg, Switzerland). Three minutes later, they underwent instillation of one drop of cyclopentolate $1 \%$ eye drops (Cyclogyl; Alcon) three times at 10-minute intervals.

Refraction and measurements of pupillary reaction and pupillary diameter were repeated nine times at 10-minute intervals, starting 30 minutes after and finishing 110 minutes after the first drop of cyclopentolate. Data obtained from the right eye were used in this study. Refraction was measured using an autorefractometer (KR-8800 Auto Kerato-Refractometer; Topcon Corporation, Tokyo, Japan).

The time of maximum cycloplegia was the primary outcome and was defined as the time point at which the spherical equivalent reached and remained within the equivalent limit $( \pm 0.25 \mathrm{D})$ of the final value at 110 minutes. In order to determine the time of maximum cycloplegia, mean differences between the spherical equivalent at each point and its final value were calculated. The time of maximum cycloplegia was determined from the time point at which the $95 \%$ confidence interval of the differences reached and remained within the equivalence limit.

The pupillary reaction and pupillary diameter were recorded by a single examiner using a slit-lamp biomicroscope (Topcon; Topcon Corporation) with a controlled fixation target, slit-lamp illumination, and room illumination. The pupillary reaction was evaluated using diffuse illumination with maximum light intensity. The pupillary diameter was measured using a $1 \mathrm{~mm}$ wide slit beam oriented directly to the patient's visual axis. The pupillary diameter was calculated by averaging the horizontal and vertical pupillary diameters when the pupil was maximally constricted.

Any serious side effects, which could have included rash development, itching or swelling (especially of the face, tongue, or throat), dizziness, trouble breathing, fainting, confusion, hallucinations, restlessness, strange behavior, and seizures, were observed and recorded.

All data were statistically analyzed by descriptive statistics. The time of maximum cycloplegia was analyzed by repeated-measures analysis of variance. The correlation between the time of maximum cycloplegia and the pupillary reaction was analyzed by Spearman's rank correlation test. All statistical analyses were performed using SPSS software, version 17.0 (SPSS Inc., Chicago, IL, USA).

\section{Results}

Sixty children (33 males, 27 females) with a mean age of 9.8 years (standard deviation [SD] 2.2; range: 5-14) were prospectively enrolled in this study. Twenty-three (38\%) had myopia with a mean refractive error of -4.00 D (SD 3.7; range: -0.75 to -15.00$)$, while $24(40 \%)$ children had hyperopia with a mean refractive error of +2.50 D (SD 2.2; range: +0.75 to +9.00$)$. All patients had brown irises. The patients' baseline characteristics are shown in Table 1.

Table I Patients' baseline characteristics

\begin{tabular}{ll}
\hline Characteristics & Mean \pm SD or $\mathbf{n}(\%)$ \\
\hline Age (years) & $9.8 \pm 2.2$ \\
Sex & \\
$\quad$ Male & $33(55)$ \\
$\quad$ Female & $27(45)$ \\
Alignment & \\
$\quad$ Orthotropia & $4 I(68)$ \\
Esotropia & $7(12)$ \\
Exotropia & $12(20)$ \\
Refractive status & \\
Emmetropia & $13(22)$ \\
Myopia & $23(38)$ \\
Hyperopia & $24(40)$ \\
\hline
\end{tabular}

Abbreviation: SD, standard deviation. 
Prior to instillation of cyclopentolate, the mean spherical equivalent was $-2.13 \mathrm{D}$ (SD 4.05). The spherical equivalents changed rapidly within the first 30 minutes after the first drop and reached $-1.34 \mathrm{D}(\mathrm{SD} 4.21)$ at 30 minutes and $-1.18 \mathrm{D}$ (SD 4.18) at 110 minutes.

Using the $95 \%$ confidence interval of the mean differences between the mean spherical equivalent at each point and its value at 110 minutes to determine the time of maximum cycloplegia, we found that maximum cycloplegia was reached 30 minutes after the first drop of cyclopentolate (Figure 1). Subgroup analysis showed that maximum cycloplegia occurred at 30 minutes in patients with emmetropia and hyperopia and at 50 minutes in patients with myopia (Figure 2).

By assessing the percentage of patients whose spherical equivalent reached and remained within $\pm 0.25 \mathrm{D}$ of their final value, we found that $87 \%(52 / 60)$ of the patients reached maximum cycloplegia 30 minutes after the first drop, while all patients reached maximum cycloplegia at 60 minutes (Figure 3).

Eight percent (5/60) of children exhibited absence of a pupillary reaction at the time of their maximum cycloplegia. The mean time to absence of a pupillary reaction was 50 minutes after the first drop of cyclopentolate (SD 10; range: 30-70), and there was a poor correlation between the time to absence of a pupillary reaction and the time of maximum cycloplegia (Spearman's rank correlation coefficient $r=-0.07$ ) (Figure 4).

The mean pupillary diameter prior to instillation of the eye drops was $2.8 \mathrm{~mm}$ (SD 2.8; range: 1.4-4.0). The mean pupillary diameter 30 minutes after the first drop was $3.7 \mathrm{~mm}$ (SD 1.0; range: 2.4-8.0), whereas the mean pupillary diameter at 110 minutes was $7.1 \mathrm{~mm}$ (SD 0.8; range: 4.5-8.0). No serious side effects were observed.

\section{Discussion}

In most children in this study, maximum cycloplegia was reached 30 minutes after the first instillation of cyclopentolate $1 \%$, with any type of strabismus, while it seemed to occur later in children with myopia. Our results support those of other studies that reported the time of the maximum cycloplegic effect of cyclopentolate. Manny et al studied the time course of cycloplegia by monitoring residual accommodation after application of one drop of cyclopentolate $1 \%$ in adults aged 18 to 29 years. ${ }^{6}$ They found that the maximum cycloplegic effect occurred at 10 minutes in individuals with light irises and at 30 to 40 minutes in individuals with dark irises. However, they only studied adults, among whom only two were Asian.

Two children in the present study had very high hyperopia (+8.50 and +9.00 D). We suspected that maximum cycloplegia may occur at a different time point in such children. The time of maximum cycloplegia in those two children with high hyperopia occurred at 30 minutes, which was not different from the other children. However, this may have been because those children had been treated with glasses. Further study is needed to determine the time of maximum cycloplegia in children with high hyperopia.

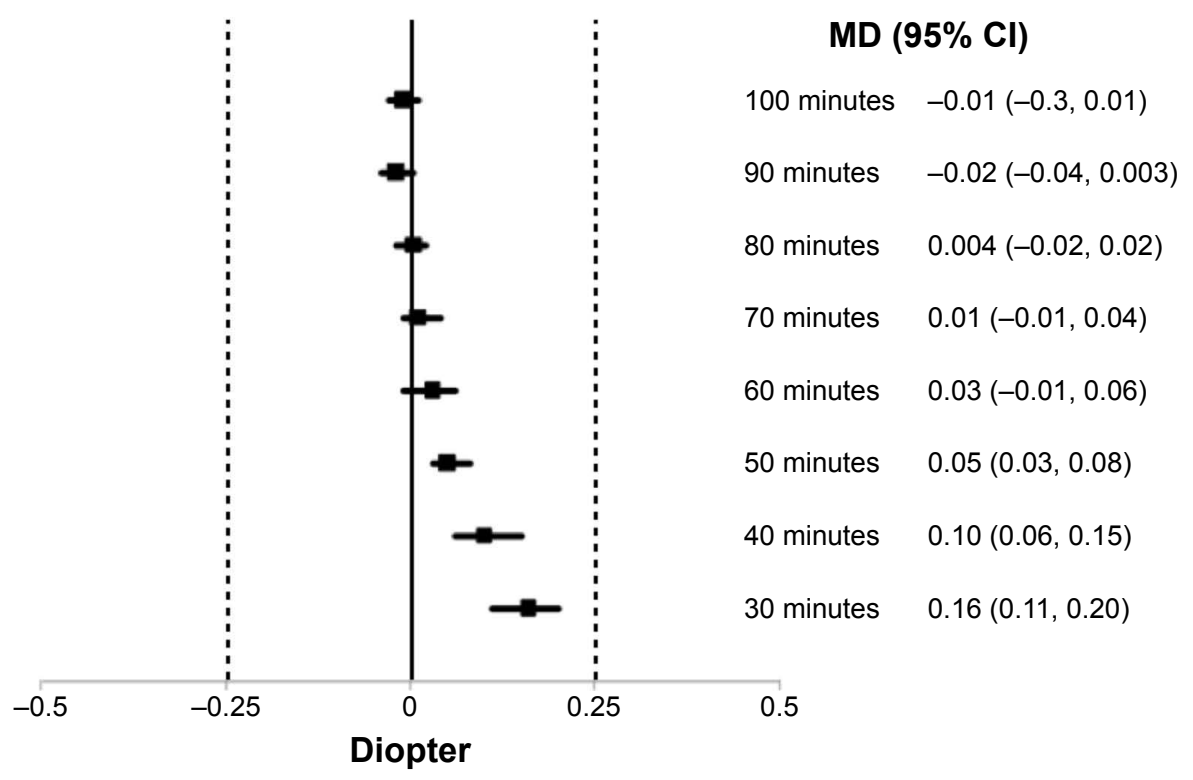

Figure I Mean differences (MD) between mean spherical equivalent at each time point and its value at II 0 minutes, and its $95 \%$ confidence interval $(\mathrm{Cl})$. 
A

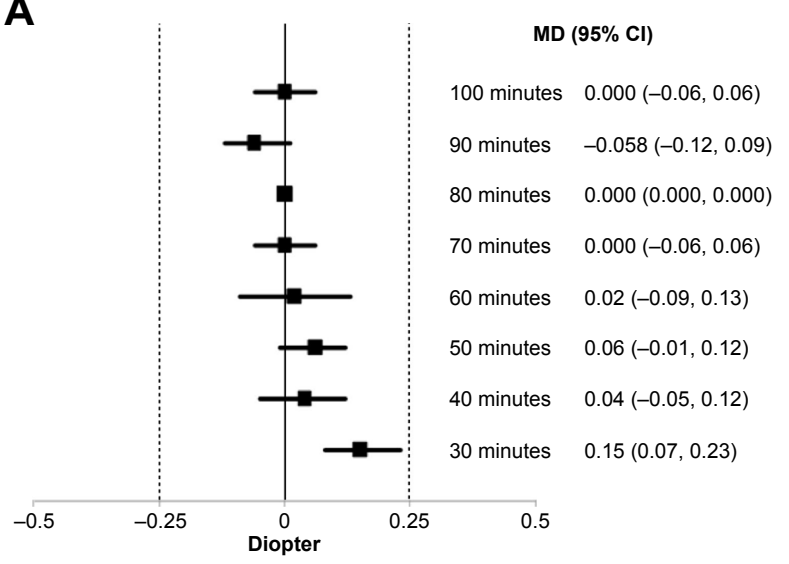

B

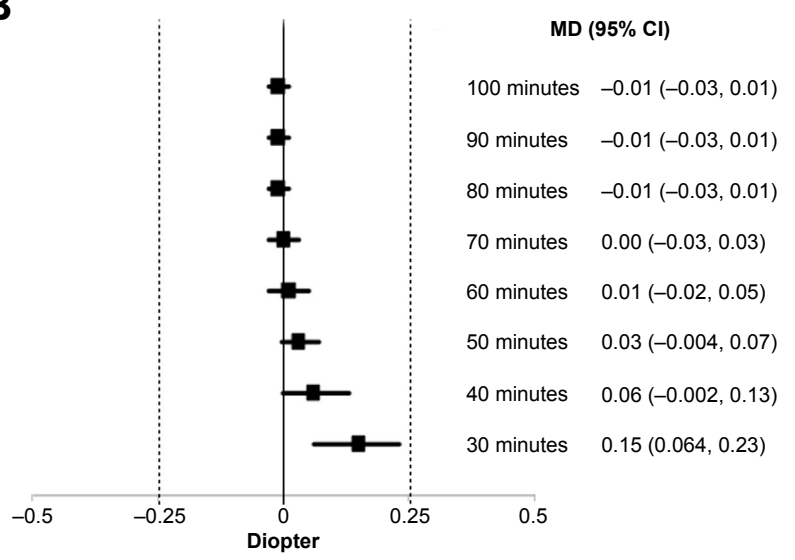

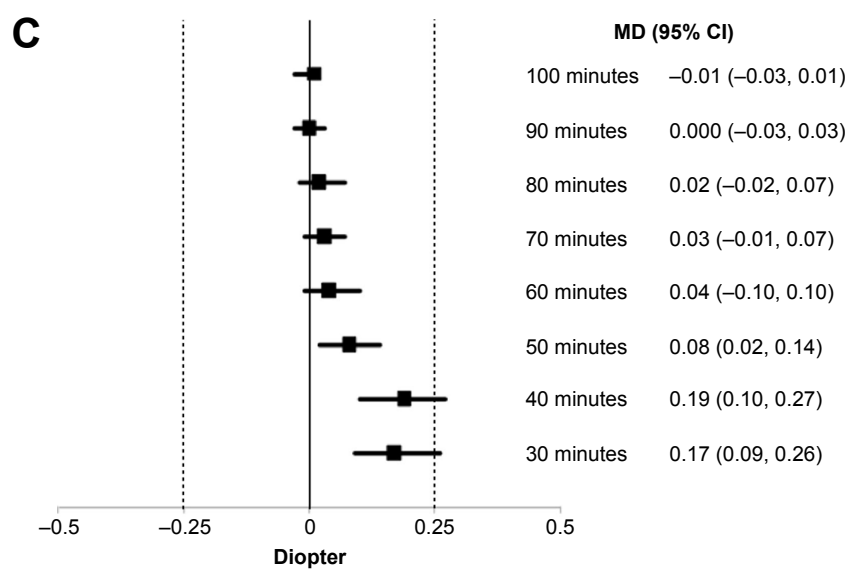

Figure 2 Mean differences (MD) between mean spherical equivalent at each time point and its value at II 0 minutes, and its $95 \%$ confidence interval (Cl), subgroup analysis for each type of refractive error.

Notes: (A) Emmetropia, (B) hyperopia, and (C) myopia.

In another study, Lin et al evaluated the cycloplegic effects of cyclopentolate and tropicamide by instilling either one to two drops of cyclopentolate $1 \%$ or tropicamide $1 \%$ twice at 5 -minute intervals in 37 Taiwanese children with myopia. ${ }^{7}$ They found that the time of maximum cycloplegia after instillation of cyclopentolate was around 45 minutes, which is comparable with our result (50 minutes). These findings support previously established clinical protocols recommending that refraction should be performed 30 to 60 minutes after instillation of cyclopentolate $1 \% .^{2-5}$ However, our analysis in myopic children showed inconclusive result at 30 and 40 minutes (Figure 2C). Therefore, further study evaluating myopic children with larger sample size should be done to confirm the result.

We found a poor correlation between the time to absence of a pupillary reaction and the time of maximum cycloplegia. Ebri et al found that only $25 \%$ of children exhibited absence of a pupillary reaction at the time of maximum cycloplegia after instillation of cyclopentolate $1 \%$, whereas $97 \%$ of children exhibited absence of a pupillary reaction after instillation of $1 \%$ atropine. ${ }^{1}$ Their results support our finding that when using cyclopentolate, only $8 \%$ of children exhibited absence of a pupillary reaction at the time of their maximum cycloplegia.

We also found that the mean pupillary diameter at the time of maximum cycloplegia was $3.7 \mathrm{~mm}$, while that at 110 minutes was $7.1 \mathrm{~mm}$. Therefore, it seems that the maximum cycloplegia was reached earlier than the maximum pupillary dilatation. This finding supports the finding of Manny et al, who reported that individuals with dark irises showed slightly less dilation than did individuals with light irises, and among all of their subjects, the time course for pupil dilation was not the same as the time course for cycloplegia. ${ }^{6}$ Additionally, Ebri et al found that $53 \%$ of children treated with cyclopentolate had a pupil diameter of 


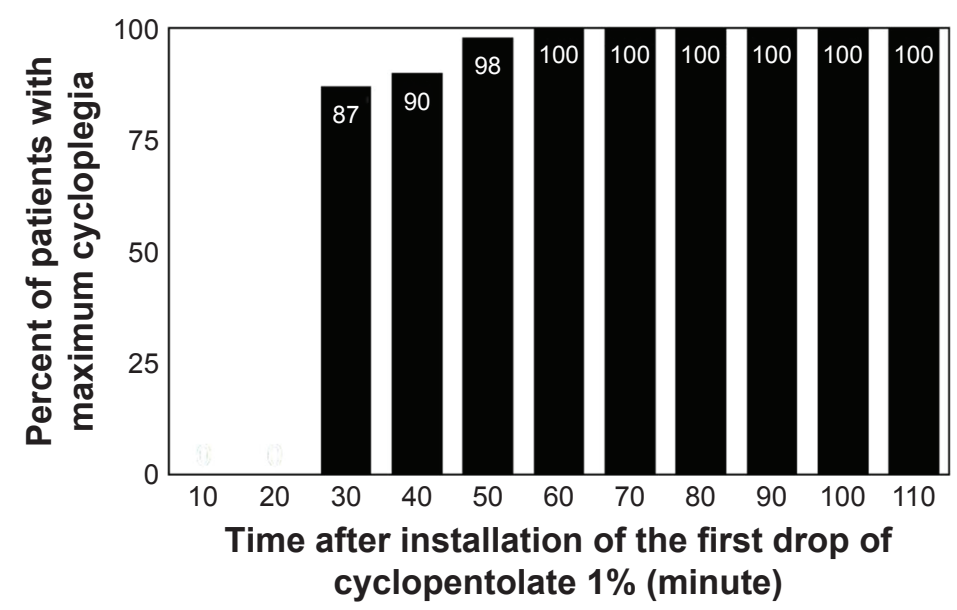

Figure 3 Percentage of patients who reached maximum cycloplegia at each time point.

$\geq 6 \mathrm{~mm}$, while all children treated with atropine had a pupil diameter of $\geq 6 \mathrm{~mm}$. $^{1}$

Importantly, most other studies that evaluated the safety and efficacy of cycloplegic agents used various regimens, such as one or two drops of cyclopentolate ${ }^{1,4,6,7,9,10}$ or a combination of cyclopentolate with tropicamide. ${ }^{1,4,11}$ The results of our study might not be applicable for the use of these other regimens.

Various techniques are available to measure accommodation, including the push-up/push-down test, minus-lenses test, focimetry, and open field autorefractor. One limitation of our study is that we used the spherical equivalent to evaluate the maximum cycloplegic effect of cyclopentolate. Therefore, the stability of the spherical equivalent might not represent complete cycloplegia. However, our subjects were children, and most available techniques of measuring accommodation were unlikely to be successful in this age group.

\section{Conclusion}

Elimination of fluctuations of accommodation or inhibition of the ciliary muscle tone improves the accuracy of refraction in children. In the present study, we found that most children with brown irises reached maximum cycloplegia within 30 minutes after the first drop of cyclopentolate 1\%. This suggests that cycloplegic refraction should be performed at least 30 minutes after instillation of cyclopentolate. We found a poor correlation between the pupillary reaction and the time of maximum cycloplegia. Our findings indicate that in clinical practice, it is not necessary to wait for absence of a pupillary reaction or maximum pupillary dilatation before

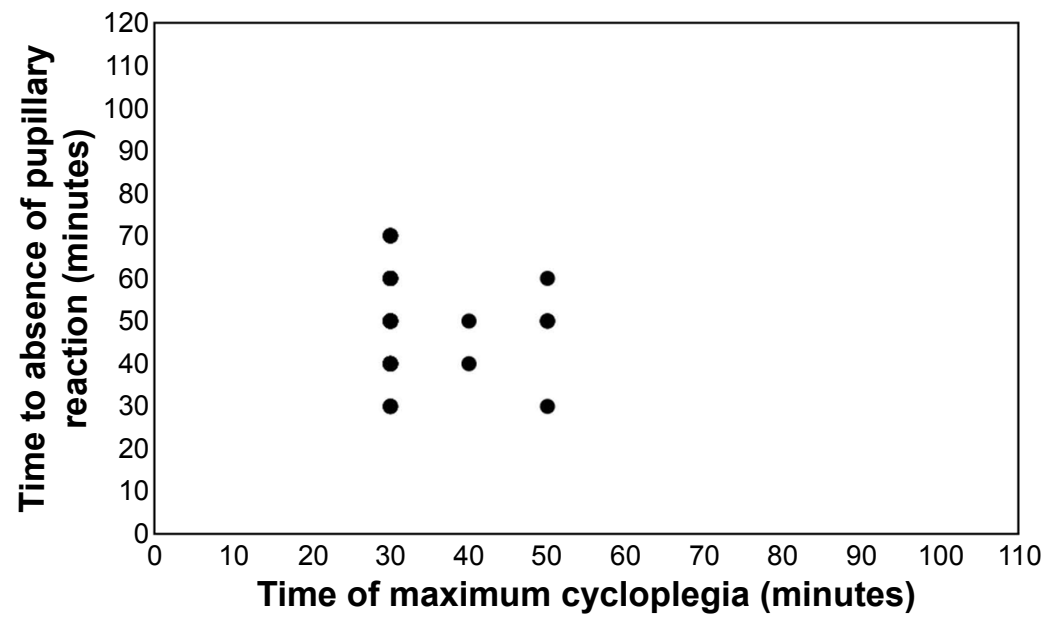

Figure 4 Relationship between time to absence of a pupillary reaction and time of maximum cycloplegia. 
performing cycloplegic refraction. The results of our study could help physicians to determine the appropriate time at which to perform cycloplegic refraction and thus shorten the patient's waiting time.

\section{Acknowledgment}

This study was supported by the Ratchadaphisek Sompoch Fund (grant number RA56/055).

\section{Disclosure}

The authors report no conflicts of interest in this work.

\section{References}

1. Ebri A, Kuper H, Wedner S. Cost-effectiveness of cycloplegic agents: results of a randomized controlled trial in Nigerian children. Invest Ophthalmol Vis Sci. 2007;48(3):1025-1031.

2. Stolzar IH. A new group of cycloplegic drugs. Am J Ophthalmol. 1953;36(1):110-112.

3. Lovasik JV. Pharmacokinetics of topically applied cyclopentolate $\mathrm{HCl}$ and tropicamide. Am J Optom Physiol Opt. 1986;63(10):787-803.
4. Miranda MN. Residual accommodation. A comparison between cyclopentolate 1 per cent and a combination of cyclopentolate 1 per cent and tropicamaide 1 per cent. Arch Ophthalmol. 1972;87(5):515-517.

5. Egashira SM, Kish LL, Twelker JD, et al. Comparison of cyclopentolate versus tropicamide cycloplegia in children. Optom Vis Sci. 1993; 70(12):1019-1026.

6. Manny RE, Fern KD, Zervas HJ, et al. 1\% Cyclopentolate hydrochloride: another look at the time course of cycloplegia using an objective measure of the accommodative response. Optom Vis Sci. 1993;70(8): 651-665.

7. Lin LL, Shih YF, Hsiao CH, et al. The cycloplegic effects of cyclopentolate and tropicamide on myopic children. $J$ Ocul Pharmacol Ther. 1998;14(4):331-335.

8. Wang X, Ma L, Wang L. Research of cyclopentolate $1 \%$ on the effect of cycloplegia for Chinese hyperopic children. Zhonghua Yan Ke Za Zhi. 2011;14(4):989-994.

9. Bagheri A, Givrad S, Yazdani S, et al. Optimal dosage of cyclopentolate $1 \%$ for complete cycloplegia: a randomized clinical trial. Eur J Ophthalmol. 2007;17(3):294-300.

10. Mohan K, Sharma A. Optimal dosage of cyclopentolate $1 \%$ for cycloplegic refraction in hypermetropes with brown irides. Indian $J$ Ophthalmol. 2011;59(6):514-516.

11. Fan DS, Rao SK, Ng JS, et al. Comparative study on the safety and efficacy of different cycloplegic agents in children with darkly pigmented irides. Clin Experiment Ophthalmol. 2004;32(5):462-467.
Clinical Ophthalmology

\section{Publish your work in this journal}

Clinical Ophthalmology is an international, peer-reviewed journal covering all subspecialties within ophthalmology. Key topics include: Optometry; Visual science; Pharmacology and drug therapy in eye diseases; Basic Sciences; Primary and Secondary eye care; Patient Safety and Quality of Care Improvements. This journal is indexed on

\section{Dovepress}

PubMed Central and CAS, and is the official journal of The Society of Clinical Ophthalmology (SCO). The manuscript management system is completely online and includes a very quick and fair peer-review system, which is all easy to use. Visit http://www.dovepress.com/ testimonials.php to read real quotes from published authors. 